\title{
Reduced amygdala reactivity and impaired working memory during dissociation in borderline personality disorder
}

\author{
Annegret Krause-Utz ${ }^{1,2,3,4} \cdot$ Dorina Winter ${ }^{1,2} \cdot$ Friederike Schriner $^{1,2}$. \\ Chui-De Chiu $^{5} \cdot$ Stefanie Lis $^{6} \cdot$ Philip Spinhoven $^{3,4,7} \cdot$ Martin Bohus $^{6}$. \\ Christian Schmahl $^{1,2} \cdot$ Bernet M. Elzinga $^{3,4}$
}

Received: 7 February 2017 / Accepted: 2 May 2017 / Published online: 19 May 2017

(c) The Author(s) 2017. This article is an open access publication

\begin{abstract}
Affective hyper-reactivity and impaired cognitive control of emotional material are core features of borderline personality disorder (BPD). A high percentage of individuals with BPD experience stress-related dissociation, including emotional numbing and memory disruptions. So far little is known about how dissociation influences the neural processing of emotional material in the context of a working memory task in BPD. We aimed to investigate whole-brain activity and amygdala functional connectivity (FC) during an Emotional Working Memory Task (EWMT) after dissociation induction in un-medicated BPD patients compared to healthy controls (HC). Using scriptdriven imagery, dissociation was induced in 17 patients ('BPD_D'), while 12 patients ('BPD_N') and $18 \mathrm{HC}$ were exposed to neutral scripts during fMRI. Afterwards, participants performed the EWMT with neutral vs. negative IAPS pictures vs. no distractors. Main outcome measures
\end{abstract}

Christian Schmahl and Bernet M. Elzinga contributed equally.

Electronic supplementary material The online version of this article (doi:10.1007/s00406-017-0806-x) contains supplementary material, which is available to authorized users.

Annegret Krause-Utz

a.d.krause@fsw.leidenuniv.nl

1 Department of Psychosomatic Medicine and Psychotherapy, Central Institute of Mental Health (CIMH), Mannheim, Germany

2 Medical Faculty, University of Heidelberg, Mannheim, Germany

3 Institute of Clinical Psychology, Leiden University, Leiden, The Netherlands

4 Leiden Institute for Brain and Cognition (LIBC), Leiden, The Netherlands were behavioral performance (reaction times, errors) and whole-brain activity during the EWMT. Psychophysiological interaction analysis was used to examine amygdala connectivity during emotional distraction. BPD patients after dissociation induction showed overall WM impairments, a deactivation in bilateral amygdala, and lower activity in left cuneus, lingual gyrus, and posterior cingulate than BPD_N, along with stronger left inferior frontal gyrus activity than HC. Furthermore, reduced amygdala FC with fusiform gyrus and stronger amygdala FC with right middle/superior temporal gyrus and left inferior parietal lobule was observed in BPD_D. Findings suggest that dissociation affects reactivity to emotionally salient material and WM. Altered activity in areas associated with emotion processing, memory, and self-referential processes may contribute to dissociative states in BPD.

Keywords Borderline personality disorder - Working memory $\cdot$ Memory $\cdot$ Neuroimaging $\cdot$ Stress

5 Department of Psychology, Centre for Cognition and Brain Studies, and Clinical and Health Psychology Centre, The Chinese University of Hong Kong, Shatin, Hong Kong

6 Institute of Psychiatric and Psychosomatic Psychotherapy, CIMH, Mannheim, Germany

7 Department of Psychiatry, Leiden University Medical Center, Leiden, The Netherlands 


\section{Introduction}

Borderline personality disorder (BPD) is a severe mental disorder, characterized by emotion dysregulation, instable cognitions, impulsivity, interpersonal disturbances, and dissociation [1-6]. Previous neuroimaging studies in BPD suggest that a hyper-reactivity and hyper-connectivity of the amygdala may underlie disturbed emotion processing in BPD [7, 8], although discrepant findings were also reported [9]. The amygdala plays a crucial role in the initiation of fear and stress responses [10] and might also be involved in stress-related dissociation [11].

Dissociation occurs in a high percentage $(\sim 75-80)$ of individuals with BPD, involving disruptions in the usually integrated functions of consciousness, perception, identity, memory, and affect and has been closely linked to psychological trauma [6,12-17]. Dissociative symptoms such as depersonalization, derealization, numbing, and analgesia may provide a state of subjective detachment from extremely stressful experiences, e.g., by dampening overwhelming emotions and reducing awareness of pain $[15,17]$. In pathological dissociation, the cost of this subjective detachment is a disruption of executive functions that are crucial to goal-directed behavior, such as attention, learning, and memory. More specifically, dissociation may hinder the conscious processing and integration of salient information in autobiographical memory, which can have detrimental effects on the development of identity and emotion regulation capacities. Dissociation may hinder the recall and learning of self-relevant information also during therapy $[13,24]$ and in BPD, dissociative symptoms predicted poor treatment outcome [18, 19]. However, the precise neuropsychological mechanisms underlying this relationship remain unclear.

Neurobiological models have linked dissociation to a dampened activity in the amygdala and increased recruitment of 'cognitive control' regions, such as the medial prefrontal cortex (mPFC), anterior cingulate cortex (ACC), and inferior frontal gyrus $[15,20]$ as well as to altered activity in the superior temporal gyrus, precuneus, posterior cingulate, which are implicated in autobiographical memory and self-referential processing [21-23]. The amygdala appears to be an important hub within this network, sharing strong functional connections with the ACC, insular and orbitofrontal cortex, mPFC, parahippocampal gyrus, precuneus, posterior cingulate, among others [24, 25]. In summary, it can be assumed that dissociation substantially affects activity within an 'amygdala network' involved in the processing of self-relevant emotional information and the initiation of stress responses. In BPD, however, so far there is little empirical evidence for this.

Only few neuroimaging studies in BPD so far investigated associations between self-reported dissociation and brain activity during experimental challenge, such as the presentation of aversive images or negative words [26-29]. To the best of our knowledge, only two neuroimaging studies in BPD used script-driven imagery to more directly investigate the effect of experimentally induced dissociation on brain activity $[21,29]$. In this well-established paradigm, a narrative of an autobiographical situation involving dissociative experiences ('dissociation script') is created and presented in an experimental setting, e.g., during functional magnetic resonance imaging (fMRI). Participants are instructed to listen to this script and to recall their autobiographical experiences as vividly as possible [23], which successfully induced dissociation in previous research $[21,29]$. When exposed to a dissociation script compared to a neutral script, BPD patients showed significantly increased activity in the left inferior frontal gyrus and diminished temporo-limbic activity, which was even more pronounced in a subgroup of traumatized patients [21]. We recently combined scriptdriven imagery with an Emotional Stroop Task (EST), to investigate the effect of a dissociation induction on interference inhibition, on a behavioral and neural level [29]. BPD patients exposed to a dissociation script showed impaired accuracy and slower reaction times for negative words than patients exposed to a neutral script. Patients after dissociation induction further showed increased left superior frontal activity in response to negative vs. neutral words [29]. However, it remains unclear how brain areas may interact during affective-cognitive processing after dissociation induction in BPD.

Moreover, to our knowledge, no study in BPD so far investigated how dissociation affects the neural processing of emotional material in the context of a working memory task, which requires the conscious manipulation of taskirrelevant stressful information. We previously used a modified version of the Emotional Working Memory Task (EWMT) in which task-irrelevant neutral vs. negative interpersonal pictures from the International Affective Picture System (IAPS) [30] or only a fixation cross (i.e., no distractors) are presented during the delay interval of a Sternberg item recognition task [27, 28]. Participants are instructed to ignore distractors, focusing solely on the WM task, thereby voluntarily inhibiting emotion processing in favor of cognitive processing. WM impairments and amygdala reactivity to negative pictures were significantly stronger in BPD patients, suggesting increased emotional distractibility compared to healthy controls (HC) [27]. During emotional distraction, BPD patients further showed a stronger coupling of the amygdala with the hippocampus and dorsomedial PFC, suggesting enhanced self-referential processing [28].

Here, we aimed to investigate the impact of experimentally induced dissociation on brain activity and amygdala 
functional connectivity during the EWMT. Studying this relationship on a behavioral and neural level might help to shed more light on the effects of stress-related dissociation in BPD. Script-driven imagery was used to induce dissociation. For patients exposed to a neutral script, we hypothesized to replicate previous findings of amygdala hyperreactivity to emotional pictures, while patients exposed to a dissociation script were expected to show significantly dampened amygdala reactivity and increased activity in frontal areas (inferior frontal gyrus, medial prefrontal cortex, anterior cingulate cortex).

\section{Materials and methods}

\section{Sample}

Sixty women aged between 18 and 45 years (40 patients with BPD according to DSM-IV [16] and 20 female HC) participated. BPD patients were recruited via advertisement on websites or referred from the residential treatment unit of the Department of Psychosomatic Medicine and Psychotherapy at the Central Institute of Mental Health (CIMH) in Mannheim, Germany. HC were recruited via newspaper advertisements. General exclusion criteria were serious somatic illnesses, traumatic brain injuries, developmental disorders, and MRI-related criteria (metal implants, pregnancy, left-handedness, claustrophobia). Exclusion criteria for $\mathrm{HC}$ were lifetime history of Axis-I/II disorders. Specific exclusion criteria for patients were psychotropic medication within 4 weeks prior to the study, substance dependence during the last year, substance abuse within 2 months prior to participation, current/lifetime psychotic or bipolarI disorder, and life-threatening suicidal crisis.

Patients were randomly assigned to two experimental conditions: 20 patients were exposed to a dissociation script ('BPD_D'), while 20 BPD patients ('BPD_N') and $20 \mathrm{HC}$ were exposed to a neutral script. An increase of $\geq 1.5$ scores on the Dissociation Stress Scale 4 (DSS-4, see below) [31] after script compared to baseline was defined as inclusion criterion for the BPD_D group (criterion was met by all participants assigned to this group). To ensure that individuals in the BPD_N group were not highly dissociated, we excluded patients with DSS-4 scores of $\geq 3$ at baseline and/or an increase of $>1.5$ scores after the experiment (three patients had to be excluded for this reason). Part of the collected data had to be discarded due to movement artifacts during fMRI (BPD_N: $n=2$, BPD_D: $n=3$, HC: $n=2$ ), technical problems during script presentation (BPD_N: $n=1$ ), or inconsistent button presses (95-100\% errors, indicated that task instructions were not understood correctly in 2 BPD_N). The final sample comprised 17 BPD_D, 12 BPD_N, and18 HC.
Clinical diagnoses were assessed by trained diagnosticians using the Structured Clinical Interview for DSM-IV Axis-I Disorders (SCID-I) [32] and International Personality Disorder Examination (IPDE) [33]. Further clinical assessment included questionnaires on symptom severity (Borderline Symptom List 23, BSL-23 [34]), childhood abuse/neglect (Childhood Trauma Questionnaire, CTQ [35]), trait dissociation (Dissociative Experiences Scale, DES [36]), depressive symptoms (Beck Depression Inventory II, BDI-II [37]), state anxiety (State Anxiety Questionnaire, STAI [38]), and Attention-Deficit Hyperactivity Disorder symptoms (childhood: Wender Utah Rating Scale, WURS [39], adulthood: ADHD-Checklist [40]).

The groups did not differ significantly regarding age and years of education (Table 1A). Both BPD groups scored significantly higher than $\mathrm{HC}$ on clinical measures but did not differ significantly from each other; all patients reported at least one type of severe to extreme childhood abuse and/ or neglect. Criteria for comorbid Posttraumatic Stress Disorder (PTSD) were met by 7 BPD_D patients (41\%) and 5 BPD_N patients (41\%), i.e., were distributed equally in both BPD groups. Further comorbidities and clinical characteristics of the two BPD groups are presented and compared in Table 1B. Dissociative states were induced using script-driven imagery and measured by the DSS-4, a self-rating scale with excellent internal consistency and reliability, high specificity, and sensitivity to change in symptomatology [31]. The DSS-4 consists of four items on current psychological (derealisation, depersonalization) and somatic (pain perception, hearing) dissociation and one item on current aversive tension (10-point Likert scales, $0=$ not at all, $9=$ extremely).

\section{Emotional Working Memory Task (EWMT)}

The EWMT was a validated Sternberg item recognition task [41], modified by Oei and colleagues [42, 43]. Our adapted version [27] consisted of 48 trials, each starting with a set of three uppercase letters (memoranda, $1000 \mathrm{~ms}$ ), followed by a delay interval $(1500 \mathrm{~ms})$, and a probe (thee uppercase letters, $2000 \mathrm{~ms}$ ). In half of the trials, one of the three memoranda was present in the probe. Participants had to press a 'yes' or 'no' button indicating whether they had recognized a target or not. During the delay interval either no distractors (only a fixation cross) or neutral vs. negative distractors (interpersonal scenes from the IAPS, selected based on arousal and valance ratings in the general population [30]) were presented. Negative pictures depicted scenes of interpersonal violence (e.g., sexual attack, physical assault, beaten/frightened child, physically mutilated body). Neutral pictures included interpersonal scenes with similar complexity (e.g., people at a market place or supermarket). Trials without distractors (only a fixation cross) 
Table 1 Demographic variables, dissociation and arousal ratings, and clinical characteristics

\begin{tabular}{|c|c|c|c|c|}
\hline (A) & BPD_D & BPD_N & $\mathrm{HC}$ & \\
\hline Age (years) & $27.41 \pm 6.20$ & $25.17 \pm 6.21$ & $29.61 \pm 8.61$ & $F_{(2,44)}=1.38, p=0.262$ \\
\hline Years of education & $10.59 \pm 2.62$ & $10.08 \pm 3.03$ & $10.72 \pm 1.99$ & $F_{(2,44)}=0.25, p=0.784$ \\
\hline \multicolumn{5}{|l|}{ DSS-4 } \\
\hline Dissociation ratings baseline & $3.44 \pm 1.99$ & $2.30 \pm 1.14$ & $1.31 \pm 0.66$ & $\begin{array}{l}F_{(2,42)}=11.27, p<0.0001 . \\
\text { BPD_D-HC: } 2.26, p<0.0001 \\
\text { BPD_N-HC: } 1.00, p=0.160 \\
\text { BPD_D-BPD_N: } 1.27, p=0.062\end{array}$ \\
\hline Dissociation ratings after script & $6.85 \pm 2.03$ & $1.85 \pm 0.84$ & $1.19 \pm 0.51$ & $\begin{array}{l}F_{(2,42)}=92.50, p<0.0001 \\
\text { BPD_D-HC: } 5.79, p<0.0001 \\
\text { BPD_N-HC: } 0.60, p=0.465 \\
\text { BPD_D-BPD_N: } 5.19, p<0.0001\end{array}$ \\
\hline Arousal rating baseline & $4.76 \pm 2.36$ & $3.91 \pm 1.97$ & $2.72 \pm 2.02$ & $\begin{array}{l}F_{(2,42)}=3.43, p=0.042 \\
\text { BPD_D-HC: } 1.90, p=0.035 \\
\text { BPD_N-HC: } 1.20, p=0.325 \\
\text { BPD_D-BPD_N: } 0.72, p=0.672\end{array}$ \\
\hline Arousal rating after script & $7.71 \pm 2.11$ & $4.50 \pm 2.65$ & $2.17 \pm 2.28$ & $\begin{array}{l}F_{(2,42)}=26.67 p<0.0001 \\
\text { BPD_D-HC: } 5.46, p<0.0001 \\
\text { BPD_N-HC: } 1.83, p=0.840 \\
\text { BPD_D-BPD_N: } 3.62, p<0.0001\end{array}$ \\
\hline $\begin{array}{l}\text { BSL-23 total score (BPD symptom } \\
\text { severity) }\end{array}$ & $47.12 \pm 19.23$ & $43.33 \pm 13.36$ & $1.33 \pm 1.81$ & $\begin{array}{l}F_{(2,44)}=60.51, p<0.0001, f^{2}=0.73 \\
\text { BPD_D-HC: } 45.78, p<0.0001 \\
\text { BPD_N-HC: } 42.00, p<0.0001 \\
\text { BPD_D-BPD_N: } 3.78, p=0.737\end{array}$ \\
\hline DES total score (trait dissociation) & $31.74 \pm 16.52$ & $26.93 \pm 13.50$ & $2.68 \pm 2.04$ & $\begin{array}{l}F_{(2,44)}=28.37, p<0.0001, f^{2}=0.56 \\
\text { BPD_D-HC: } 29.01, p<0.0001 \\
\text { BPD_N-HC: } 24.26, p<0.0001 \\
\text { BPD_D-BPD_N: } 4.81, p=0.547\end{array}$ \\
\hline BDI-II (depressive symptoms) & $24.47 \pm 11.89$ & $26.75 \pm 10.68$ & $1.67 \pm 2.25$ & $\begin{array}{l}F_{(2,44)}=38.49, p<0.0001, f^{2}=0.64 \\
\text { BPD_D-HC: } 22.80, p<0.0001 \\
\text { BPD_NHC: } 25.08, p<0.0001 \\
\text { BPD_D-BPD_N: } 2.28, p=0.783\end{array}$ \\
\hline STAI state ${ }^{\mathrm{a}}$ (state anxiety) & $56.19 \pm 10.13$ & $52.92 \pm 6.36$ & $29.39 \pm 5.41$ & $\begin{array}{l}F_{(2,43)}=54.90, p<0.0001, f^{2}=0.74 \\
\text { BPD_D-HC: } 26.79, p<0.0001 \\
\text { BPD_N-HC: } 23.53, p<0.0001 \\
\text { BPD_D-BPD_N: } 2.55, p=0.503\end{array}$ \\
\hline STAI trait ${ }^{\mathrm{a}}$ (trait anxiety) & $58.13 \pm 7.03$ & $60.58 \pm 5.83$ & $28.72 \pm 4.66$ & $\begin{array}{l}F_{(2,43)}=138,83, p<0.0001, f^{2}=0.87 \\
\text { BPD_D-HC: } 29.40, p<0.0001 \\
\text { BPD_N-HC: } 31.86, p<0.0001 \\
\text { BPD_D-BPD_N: } 2.05, p=0.522\end{array}$ \\
\hline WURS (childhood ADHD symptoms) & $98.80 \pm 41.16$ & $94.42 \pm 27.91$ & $49.53 \pm 27.52$ & $\begin{array}{l}F_{(2,39)}=9.88, p<0.0001, f^{2}=0.39 \\
\text { BPD_D-HC: } 49.27, p<0.0001 \\
\text { BPD_N -HC: } 44.88, p<0.0001 \\
\text { BPD_D-BPD_N: } 4.39, p=0.938\end{array}$ \\
\hline $\begin{array}{l}\text { ADHD checklist }{ }^{\mathrm{a}} \text { (adult ADHD symp- } \\
\text { toms) }\end{array}$ & $14.94 \pm 9.80$ & $16.83 \pm 8.33$ & $3.94 \pm 2.88$ & $\begin{array}{l}F_{(2,44)}=14.11, p<0.0001, f^{2}=0.39 \\
\text { BPD_D-HC: } 10.99, p<0.0001 \\
\text { BPD_N-HC: } 12.89, p<0.0001 \\
\text { BPD_D-BPD_N: } 1.89, p=0.789\end{array}$ \\
\hline $\begin{array}{l}\text { CTQ total sum-score (childhood abuse } \\
\text { and neglect) }\end{array}$ & $68.23 \pm 25.12$ & $70.58 \pm 16.46$ & $33.39 \pm 11.88$ & $\begin{array}{l}F_{(2,44)}=20.34, p<0.0001, f^{2}=0.48 \\
\text { BPD_D-HC: } 34.91, p<0.0001 \\
\text { BPD_N-HC: } 37.19, p<0.0001 \\
\text { BPD_D-BPD_N: } 2.29, p=0.944\end{array}$ \\
\hline \multicolumn{2}{|c|}{ (B) Clinical characteristics and comorbidities $n(\%)$} & BPD_D $(n=17)$ & BPD_N $(n=12)$ & $\chi^{2}$ tests \\
\hline BPD criteria fulfilled & (DSM-IV) & & & \\
\hline Fear of abandonment & 1 & $9(53 \%)$ & $12(100 \%)$ & $\chi^{2}=0.37, p=0.830$ \\
\hline Instable relationships & 2 & $8(47 \%)$ & $8(67 \%)$ & $\chi^{2}=1.60, p=0.450$ \\
\hline Identity disturbance & 3 & $10(59 \%)$ & $10(83 \%)$ & $\chi^{2}=3.53, p=0.171$ \\
\hline
\end{tabular}


Table 1 continued

\begin{tabular}{|c|c|c|c|c|}
\hline \multicolumn{2}{|c|}{ (B) Clinical characteristics and comorbidities $n(\%)$} & \multirow{2}{*}{$\begin{array}{c}\text { BPD_D }(n=17) \\
7(41 \%)\end{array}$} & \multirow{2}{*}{$\frac{\text { BPD_N }(n=12)}{5(42 \%)}$} & \multirow{2}{*}{$\begin{array}{l}\chi^{2} \text { tests } \\
\chi^{2}=0.52, p=0.773\end{array}$} \\
\hline Impulsivity & 4 & & & \\
\hline Non-suicidal self-injury & 5 & $14(82 \%)$ & $8(67 \%)$ & $\chi^{2}=1.08, p=0.583$ \\
\hline Affective instability & 6 & $17(100 \%)$ & $12(100 \%)$ & - \\
\hline Emptiness & 7 & $12(71 \%)$ & $9(75 \%)$ & $\chi^{2}=0.73, p=0.695$ \\
\hline Anger & 8 & $17(100 \%)$ & $8(67 \%)$ & $\chi^{2}=2.31, p=0.316$ \\
\hline Dissociation & 9 & $17(100 \%)$ & $12(100 \%)$ & - \\
\hline Self-injurious behavior (last 12 & & $15(88 \%)$ & $10(83 \%)$ & $\chi^{2}=0.14, p=0.706$ \\
\hline \multirow[t]{2}{*}{ Major depressive disorder } & Current & $2(12 \%)$ & $0(0 \%)$ & $\chi^{2}=1.49 p=0.223$ \\
\hline & Lifetime & $15(88 \%)$ & $8(66 \%)$ & $\chi^{2}=2.28, p=0.131$ \\
\hline Dysthymia & Current & $0(0 \%)$ & $1(8 \%)$ & $\chi^{2}=1.51, p=0.219$ \\
\hline \multirow[t]{2}{*}{ Panic disorder } & Current & $3(18 \%)$ & $2(17 \%)$ & $\chi^{2}=0.01, p=0.970$ \\
\hline & Lifetime & $5(29 \%)$ & $3(18 \%)$ & $\chi^{2}=0.05, p=0.824$ \\
\hline \multirow[t]{2}{*}{ Social phobia } & Current & $8(47 \%)$ & $2(17 \%)$ & $\chi^{2}=2.83, p=0.093$ \\
\hline & Lifetime & $10(59 \%)$ & $4(33 \%)$ & $\chi^{2}=1 / 78, p=0.182$ \\
\hline \multirow[t]{2}{*}{ Specific phobia } & Current & $3(18 \%)$ & $1(8 \%)$ & $\chi^{2}=0.48, p=0.488$ \\
\hline & Lifetime & $3(18 \%)$ & $1(8 \%)$ & $\chi^{2}=0.48, p=0.488$ \\
\hline Obsessive compulsive disorder & Lifetime & $4(24 \%)$ & $1(8 \%)$ & $\chi^{2}=1.09, p=0.296$ \\
\hline \multirow[t]{2}{*}{ Posttraumatic stress disorder } & Current & $7(41 \%)$ & $5(41 \%)$ & - \\
\hline & Lifetime & $8(47 \%)$ & $5(41 \%)$ & $\chi^{2}=0.88, p=0.646$ \\
\hline Somatization disorder & Lifetime & $1(6 \%)$ & $0(0 \%)$ & $\chi^{2}=0.71, p=0.398$ \\
\hline \multirow[t]{2}{*}{ Eating disorders } & Current & $1(6 \%)$ & $1(8 \%)$ & - \\
\hline & Lifetime & $7(41 \%)$ & $3(18 \%)$ & $\chi^{2}=0.76, p=0.384$ \\
\hline Drug abuse & Lifetime & $2(12 \%)$ & $1(8 \%)$ & $\chi^{2}=0.15, p=0.929$ \\
\hline Alcohol abuse & Lifetime & $1(6 \%)$ & $0(0 \%)$ & $\chi^{2}=1.52, p=0.468$ \\
\hline Previous medication & & $13(76 \%)$ & $9(75 \%)$ & $\chi^{2}=0.008, p=0.927$ \\
\hline Acamprosate & & $0(0 \%)$ & $1(8 \%)$ & \\
\hline Atypical antipsychotics & & $1(6 \%)$ & $1(8 \%)$ & \\
\hline BZD & & $2(12 \%)$ & $1(8 \%)$ & $\chi^{2}=6.21, p=0.400$ \\
\hline SNRI & & $3(18 \%)$ & $2(17 \%)$ & \\
\hline SSRI & & $6(35 \%)$ & $1(8 \%)$ & \\
\hline TCA & & $1(6 \%)$ & $3(18 \%)$ & \\
\hline \multicolumn{5}{|l|}{ Time of last medication ${ }^{\mathrm{b}}$} \\
\hline 1 month ago & & $3(18 \%)$ & $1(8 \%)$ & \\
\hline$\geq 3$ month ago & & $2(12 \%)$ & $1(8 \%)$ & \\
\hline$\geq 6$ month ago & & $2(12 \%)$ & $6(50 \%)$ & $\chi^{2}=4.76, p=0.190$ \\
\hline$\geq 12$ month ago & & $4(24 \%)$ & $1(8 \%)$ & \\
\hline
\end{tabular}

$M$ mean, $S D$ standard deviation, $D S S$-4 Dissociation Stress Scale 4, BPD_D patients with borderline personality disorder exposed to a dissociation script, $B P D \_N$ patients with borderline personality disorder exposed to a neutral script, $H C$ healthy controls, $B S L-23$ borderline, $B Z D$ benzodiazepine, SSRI selective serotonin reuptake inhibitor, SNRI serotonin-norepinephrine reuptake inhibitor, TCA Tricyclic antidepressant. Symptom List 23, DES Dissociative Experience Scale, BDI Beck Depression Inventory, STAI State Anxiety Inventory, CTQ Childhood Trauma Questionnaire, WURS Wender Utah Rating Scale

a STAI scores in one BPD_D patient and WURS scores in 3 HC and 2 BPD_D patients were missing

b Information in 2 BPD_D patients was missing 
were added, as even neutral interpersonal stimuli were found to be perceived as emotionally arousing in individuals with BPD, increasing amygdala activity [27]. In addition, participants performed 15 trials of the basic Sternberg paradigm without distractors (i.e., only a fixation cross) to assess baseline working memory. Target-present and targetabsent trials were equal in all conditions and balanced in a pseudo-random manner. Software Presentation (Neurobehavioural systems, http://www.neurobs.com/) was used to present stimuli and record behavioral data.

\section{Procedure}

The experiment was approved by the local ethics committee (Medical Faculty of Heidelberg University) and conducted at the CIMH in Mannheim, Germany. All participants received information about the study and scanning procedure, signed written informed consent, and underwent diagnostic and clinical assessment. Then, participants prepared a personalized script of 30-s length together with one experimenter (F.S. and D.W.). Patients assigned to the BPD_D group were instructed to report a non-traumarelated autobiographical situation involving dissociation. BPD_N and HC were instructed to report an emotionally neutral everyday situation. A person unknown to participants read each script aloud recording it on audio tape. During the experiment, participants first practiced five trials of the EWMT outside the scanner. Inside the scanner, scripts were presented via headphones. DSS-4 ratings were assessed before and after scripts. Then participants performed the EWMT (first the 15 trials of the basic Sternberg paradigm, then the EWMT with and without distractors). Participants were instructed to focus on the middle of the screen, to concentrate on the task only and to ignore distractors. Event-related fMRI data were acquired during ratings, script, and EWMT.

\section{FMRI scan protocol}

MRI was conducted using a 3-Tesla Siemens TRIO-Scanner (Siemens, Erlangen). Head cushions and headphones were used to reduce head movement artifacts and scanning noise. Blood oxygen level-dependent (BOLD) signal was measured with 36 3-mm transversal slices covering the entire brain using gradient echo-planar-imaging (EPI) [T2-weighted contrast, field of view $=192 \times 192 \mathrm{~mm}$, voxel size $=3 \times 3 \times 3 \mathrm{~mm}^{3}$, voxel matrix $=64 \times 64$, flip angle $=80^{\circ}$, spin-echo time $=30 \mathrm{~ms}$, inter-scan repetition time $(\mathrm{TR})=2000 \mathrm{~ms}$. After fMRI, as individual template for functional data, a high-resolution anatomical scan was acquired using three-dimensional magnetization-prepared rapid acquisition gradient echo (MPRAGE) [T1-weighted contrast, voxel size $\left.=1 \times 1 \times 1 \mathrm{~mm}^{3}\right]$.

\section{Statistical analysis}

Custom statistical software (SPSS, Chicago: SPSS Inc) was used for manipulation check, behavioral data analysis, and follow-up (subgroup) comparisons. Normal distribution was checked for all variables using the Kolmogorov-Smirnov test. For repeated measurement analysis of variance (rmANOVA), assumptions of variance equality (Levene's tests) and sphericity (Mauchly's test) were checked (in case of violations Greenhouse-Geisser corrections were applied). Significant effects were followed up using between-group or paired $t$ tests $(p<0.05$, two tailed).

\section{Manipulation check}

A $3 \times 2$ rmANOVA with DSS4-scores before and after script as dependent variables (within-subject factor Time) and Group as between-subject factor was performed to check whether self-reported dissociation significantly changed after script.

\section{Behavioral (WM) data}

WM data were checked for outliers. Errors were scored as incorrect, too early responses, and misses (omissions) separately. Percentage of incorrect responses as well as reaction times (RTs) for correct trials were analyzed using two separate $3 \times 3$ rmANOVAs with Group as betweensubjects factor and Condition (no distraction vs. neutral vs. negative distractors) as within-subject factor. Differences in specific error types (wrong responses, too early responses, misses) were evaluated using a multivariate ANOVA (MANOVA) with Group as fixed factor. Basic working memory performance (errors, RTs) of trials without distraction was compared between groups using two separate ANOVAs.

\section{Fmri data}

Functional imaging data were analyzed using standard procedures implemented in the Statistical Parametric Mapping package (SPM8, Neurobehavioral systems, Berkeley, CA; http://www.fil.ion.ucl.ac.uk/spm/). EPI time series were preprocessed according to common standards, including slice time correction, spatial realignment, and unwarping to correct for head motion, co-registration onto participants' high-resolution $\mathrm{T} 1 \mathrm{scan}$, normalization to the standard brain of the Montreal Neurological Institute (MNI) space, and smoothing using a Gaussian kernel with a full width at half maximum (FWHM) of $9 \mathrm{~mm}$. Statistical analyses of our event-related design relied upon the general linear model (GLM) to estimate effects of interest [44]. 


\section{Region of interest (ROI) and whole-brain (WB) analysis}

Single subject level For each participant, task-related activity was identified by convolving a vector of the onset times of the following seven experimental events of interest with a canonical hemodynamic response: memoranda, delay intervals (no, neutral, negative distractors), and probes after no, neutral, and negative distractors, respectively. The GLM further included nuisance variables to control for movement artifacts.

Group level To test our a priori hypothesis of decreased amygdala activity in BPD_D, a ROI analysis was conducted using an anatomical mask of the bilateral amygdala (created by the Automated Anatomical Labeling software, AAL [45]), smoothed with a cube of voxels of size (FWHM) of $9 \mathrm{~mm}$. Values of percent signal change in this region during delay intervals (no vs. neutral vs. negative distractors) were extracted for each participant using the rfxplot toolbox [46] and exported to SPSS. Equivalent to the analysis of behavioral data, a $3 \times 3$ rmANOVA (between-subject factor: Group, within-subject factor: Condition) was then performed in SPSS. To ensure that group differences were not confounded by basic differences in arousal or WM, we repeated the analysis with arousal ratings as well as WM errors as covariate, using two separate rmANCOVAs.

WB analysis Consistent with our previous studies [27, 28], a full factorial design was used to model effects of group and experimental task. Within this model, we tested for overall group differences (F contrast) during negative distractors relative to no distractors (as a more neutral control condition [47-49]). Gaussianized $F / T$ statistic images were determined using a significance threshold of $p<0.05$, Family-wise error (FWE) corrected for multiple comparisons on the voxel-wise WB level. Based on our a priori hypotheses, small volume corrections (SVC) with pre-defined anatomical masks of the inferior frontal gyrus, mPFC, and ACC (regions of interest) were applied. To follow-up significant WB group effects in subgroup comparisons, parameter estimates were exported to SPSS, and analyzed using between-group $t$ tests $(p<0.05)$.

\section{Psychophysiological interaction analysis (PPI) analysis}

The generalized PPI (gPPI) toolbox by McLaren [50] was applied to analyze changes in the correlation of time series of the amygdala (seed region) with time series of regions across the whole brain, dependent on our experimental manipulation $[51,52]$. For the amygdala seed, the same anatomical mask of bilateral amygdala and the same contrast (negative vs. no distractors) as in the above-mentioned ROI analyses were used. For each participant, mean time series of activity from voxels falling within this anatomical mask were extracted and first-level contrasts for the EWMT conditions were computed. Since PPI analysis of event-related designs lacks power [52], increasing the probability of false-negative results (Type-II-error), we decided to apply a more lenient initial clustering threshold of $p<0.001$, uncorrected on the voxel-wise level (cluster size $k>10, Z>3.5$ ). However, only clusters FWE corrected for multiple comparisons $(p<0.05)$ at the cluster level are discussed. PPI beta estimates of significant clusters for negative vs. no distractors (F contrast) were extracted and exported to SPSS. Overall group differences were then evaluated with a MANOVA and followed up using post hoc $t$ tests. To ensure that group differences were not confounded by basic differences in WM, we repeated the analysis with WM errors as covariate (MANCOVA).

\section{Results}

\section{Dissociation induction}

Means with standard deviation of DSS-4 scores are reported in Table 1A. Main effects of Time $\left(F_{(1.43}\right)=23.01$, $\left.p<0.0001, \eta^{2}=0.35\right)$ and $\operatorname{Group}\left(F_{(2,43)}=48.57, p<0.0001\right.$, $\left.\eta^{2}=0.69\right)$ and the interaction effect $\left(F_{(2,43)}=43.79\right.$, $\left.p<0.0001, \eta^{2}=0.67\right)$ were significant with higher scores after script than baseline in BPD_D $\left(t_{(16)}=7.57, p<0.0001\right)$ but not in the other groups $(p>0.05)$.

\section{Behavioral data}

There were no significant group differences in basic WM (without distractors, $p>0.05$, data not shown). Figure 1 shows means \pm standard errors of the mean (SEM) for percentage of incorrect responses (Fig. 1a) and RTs of correct trials (Fig. 1b) during the EWMT in BPD_D, BPD_N, and HC.

\section{Errors during the EWMT}

The rmANOVA revealed a significant Group effect $\left(F_{(2,43)}=4.43, p=0.018, \eta^{2}=0.17\right)$ with an overall higher percentage of incorrect responses in BPD_D than in BPD_N $(p=0.012)$ and in HC $(p=0.019)$ (see Fig. 1a). The MANOVA further indicated that there were significant group differences in the number of misses $\left(F_{(2,43)}=6.86\right.$, $\left.p=0.003, \eta^{2}=0.24\right)$, due to more misses in BPD_D than in BPD_N $(p=0.001)$ and $\mathrm{HC}(p=0.011)$, as shown in Supplemental Figure S1.

\section{Reaction times during the EWMT}

The rmANOVA revealed a significant Condition effect $\left(F_{(2,42)}=4.17, p=0.022, \eta^{2}=0.17\right)$ with longer RTs during neutral $(p=0.019)$ and negative distractors $(p=0.003)$ than during no distractors, but no significant Group effect or interaction effect (both $p>0.05$ ) (see Fig. 1b). 

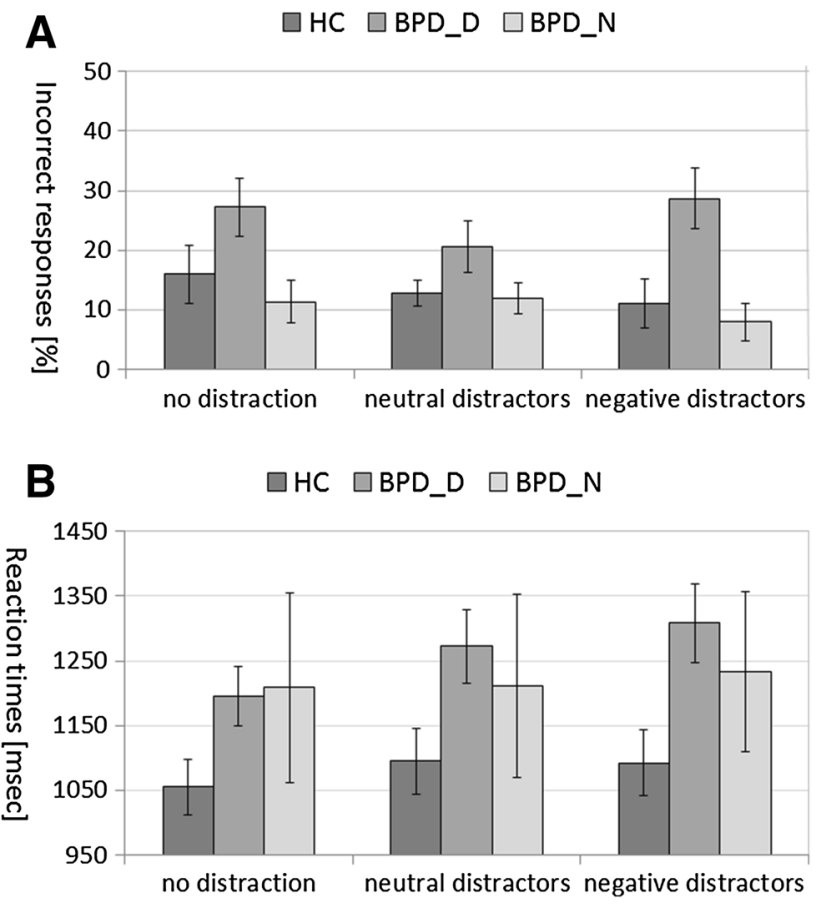

Fig. 1 Working memory performance during the Emotional Working Memory Task (after no distraction, after neutral distractors, after negative distractors) in patients with borderline personality disorder (BPD) after dissociation induction (BPD_D) and after the neutral script (BPD_N) as well as in healthy controls (HC). a Means \pm standard errors of the mean of percentage of errors. $\mathbf{b}$ Means \pm standard errors of the mean of reaction times in correct trials

\section{FMRI data}

\section{ROI analysis}

Figure 2 depicts mean \pm SEM of percent signal change in the bilateral amygdala. The rmANOVA revealed a significant main effect for Group $\left(F_{(2,44)}=5.36, p=0.008\right.$, $\left.f^{2}=0.20\right)$ with higher amygdala activity in BPD_N than in BPD_D $(p=0.002)$ and in HC $(p=0.023)$ (no significant differences between BPD_D and HC, $p>0.05$ ). Furthermore, there was a trend for a main effect of Condition $\left(F_{(2,87)}=3.21, p=0.050, f^{2}=0.13\right)$ (interaction effect: $p>0.05)$.

When including self-reported aversive tension (DSS-4 item) as covariate, group differences remained significant $\left(F_{(2,44)}=4.89, p=0.012, f^{2}=0.19\right)$. Likewise, the rmANCOVA with WM errors as covariate still revealed a significant Group effect $\left(F_{(2,42)}=3.43, p=0.042, f^{2}=0.14\right)$ with higher amygdala activity in BPD_N than in BPD_D $(p=0.015)$ and $\mathrm{HC}(p=0.043)$.

\section{Whole-brain analysis}

As a main effect of task ( $F$ contrast), there were significant changes in brain activity in the bilateral amygdala, hippocampus, insula, cingulate gyrus, dorsomedial, dorsolateral, ventrolateral prefrontal, occipital, parietal, temporal, and subcortical regions (see Table 2). Significant group

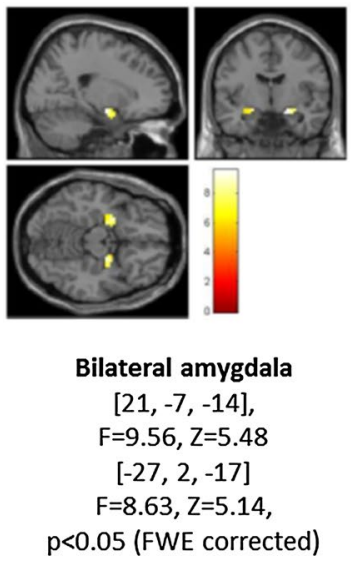

Fig. 2 Percent signal change in the bilateral amygdala (region of interest analysis) during the Emotional Working Memory Task (no distraction, neutral distractors, negative distractors) in patients with borderline personality disorder (BPD) after dissociation induction
(BPD_D) and after the neutral script (BPD_N) as well as in healthy controls (HC). Clusters in the bilateral amygdala, detected by the main effect of task $(p<0.05$, FWE corrected on the voxel-wise level) are depicted on the left 
Table 2 Results of the full factorial model of brain activity during the Emotional Working Memory Task

\begin{tabular}{|c|c|c|c|c|c|c|c|c|}
\hline F Contrast & $\begin{array}{l}\text { Brain region: label } \\
\text { (Brodmann area) }\end{array}$ & Lobe & & Cluster size & $\begin{array}{l}\text { Peak voxel coor- } \\
\text { dinates (MNI: } X \text {, } \\
Y, Z)\end{array}$ & $F$ value & $Z$ value & $p$ value \\
\hline \multirow{28}{*}{$\begin{array}{l}\text { Main effect of } \\
\text { condition }\end{array}$} & Fusiform gyrus & Occipital Lobe & N.A. & 6225 & $30-58-14$ & 31.67 & Inf & $p(\mathrm{FWE})<0.001$ \\
\hline & Fusiform gyrus & Temporal Lobe & BA 20 & 6225 & $36-43-20$ & 29.10 & Inf & $p(\mathrm{FWE})<0.001$ \\
\hline & Fusiform gyrus & Temporal Lobe & BA 37 & 6225 & $42-49-17$ & 28.92 & Inf & $p(\mathrm{FWE})<0.001$ \\
\hline & Postcentral gyrus & Parietal Lobe & BA 3 & 246 & $-39-2252$ & 16.36 & 7.45 & $p(\mathrm{FWE})<0.001$ \\
\hline & Middle frontal gyrus & Frontal Lobe & BA 6 & 246 & $-24-452$ & 12.14 & 6.31 & $p(\mathrm{FWE})<0.001$ \\
\hline & Cingulate gyrus & Limbic Lobe & BA 32 & 390 & -61146 & 16.11 & 7.39 & $p(\mathrm{FWE})<0.001$ \\
\hline & Medial Frontal Gyrus & Frontal Lobe & BA 6 & 390 & $-6-455$ & 12.61 & 6.45 & $p(\mathrm{FWE})<0.001$ \\
\hline & Middle Frontal Gyrus & Frontal Lobe & BA 32 & 390 & 91149 & 12.14 & 6.32 & $p(\mathrm{FWE})<0.001$ \\
\hline & Insula & Sub-lobar & BA 13 & 99 & -30234 & 14.92 & 7.09 & $p(\mathrm{FWE})<0.001$ \\
\hline & Inferior Frontal Gyrus & Frontal Lobe & BA9 & 173 & -54831 & 13.44 & 6.69 & $p(\mathrm{FWE})<0.001$ \\
\hline & Inferior Frontal Gyrus & Frontal Lobe & BA9 & 173 & -45531 & 12.51 & 6.42 & $p(\mathrm{FWE})<0.001$ \\
\hline & Middle Frontal Gyrus & Frontal Lobe & BA46 & 173 & -482325 & 7.48 & 4.68 & $p(\mathrm{FWE})=0.029$ \\
\hline & Insula & Sub-lobar & BA 13 & 110 & 36207 & 12.22 & 6.34 & $p(\mathrm{FWE})<0.001$ \\
\hline & $\begin{array}{l}\text { Dorsolateral prefron- } \\
\text { tal cortex }\end{array}$ & $?$ & BA 9 & 104 & 45531 & 12.18 & 6.33 & $p(\mathrm{FWE})<0.001$ \\
\hline & Putamen & Sub-lobar & Putamen & 68 & $-188-2$ & 12.04 & 6.29 & $p(\mathrm{FWE})<0.001$ \\
\hline & Amygdala & Limbic Lobe & Amygdala & 68 & $-272-17$ & 8.63 & 5.14 & $p(\mathrm{FWE})=0.004$ \\
\hline & Middle Frontal Gyrus & Frontal Lobe & BA 6 & 58 & $30-452$ & 11.06 & 5.98 & $p(\mathrm{FWE})<0.001$ \\
\hline & $\begin{array}{l}\text { Inferior Parietal } \\
\text { Lobule }\end{array}$ & Parietal Lobe & BA 40 & 91 & $-48-6440$ & 11.05 & 5.98 & $p(\mathrm{FWE})<0.001$ \\
\hline & Putamen & Sub-lobar & Putamen & 40 & 2184 & 10.83 & 5.91 & $p(\mathrm{FWE})<0.001$ \\
\hline & Inferior Frontal Gyrus & Frontal Lobe & BA 47 & 85 & $-4226-14$ & 10.57 & 5.82 & $p(\mathrm{FWE})<0.001$ \\
\hline & Amygdala & Limbic Lobe & Amygdala & 65 & $21-7-14$ & 9.56 & 5.48 & $p(\mathrm{FWE})=0.001$ \\
\hline & Hippocampus & Sub-lobar & Hippocampus & 65 & $30-10-17$ & 9.02 & 5.28 & $p(\mathrm{FWE})=0.002$ \\
\hline & $\begin{array}{l}\text { Superior temporal } \\
\text { gyrus }\end{array}$ & Temporal Lobe & BA 22 & 8 & $63-44$ & 8.27 & 5.00 & $p(\mathrm{FWE})=0.007$ \\
\hline & Precuneus & Parietal Lobe & BA 7 & 20 & $-24-5849$ & 8.21 & 4.98 & $p(\mathrm{FWE})=0.008$ \\
\hline & Medial frontal gyrus & Frontal Lobe & BA 10 & 9 & $-350-5$ & 7.89 & 4.85 & $p(\mathrm{FWE})=0.014$ \\
\hline & Inferior frontal gyrus & Frontal Lobe & BA 46 & 6 & -452916 & 7.79 & 4.81 & $p(\mathrm{FWE})=0.016$ \\
\hline & $\begin{array}{l}\text { Superior temporal } \\
\text { gyrus }\end{array}$ & Temporal Lobe & BA 38 & 5 & $4520-23$ & 7.79 & 4.81 & $p(\mathrm{FWE})=0.016$ \\
\hline & Hippocampus & Limbic Lobe & Hippocampus & 5 & $-30-16-17$ & 7.47 & 4.68 & $p(\mathrm{FWE})=0.029$ \\
\hline \multirow{6}{*}{$\begin{array}{l}\text { Main effect } \\
\text { of Group } \\
\text { (F contrast) } \\
\text { negative } \\
\text { distractors } \\
\text { relative to no } \\
\text { distraction }\end{array}$} & Cuneus & Occipital Lobe & BA18 & 247 & $-3-7922$ & 13.88 & 4.63 & $p(\mathrm{FWE})=0.031$ \\
\hline & Lingual Gyrus & Occipital Lobe & BA19 & & $-15-61-5$ & 10.65 & 3.97 & \\
\hline & Posterior Cingulate & Limbic Lobe & BA30 & & $-15-644$ & 9.34 & 3.67 & \\
\hline & Inferior Frontal Gyrus & Frontal Lobe & BA9 & 102 & -48528 & 12.08 & 4.27 & $p(\mathrm{FWE})=0.010^{*}$ \\
\hline & Inferior Frontal Gyrus & Frontal Lobe & BA44 & & -54819 & 11.08 & 4.07 & \\
\hline & Insula & Sub-Lobar & BA13 & & -421119 & 7.92 & 3.32 & \\
\hline
\end{tabular}

All $z$ values were determined by an initial cluster-forming threshold of $p<0.05$ family-wise error (FWE) corrected on a whole-brain voxel-wise level. Clusters detected after small volume correction (SVC) $(p<0.05)$ are indicated by an asterisk $(*)$ 
differences for brain activity during negative vs. no distractors were found for a cluster comprising left cuneus, lingual gyrus, and posterior cingulate (whole-brain, FWEcorrected $p<0.05$ ) and in the left inferior frontal gyrus (BA44) and insula (BA13) (after SVC with the IFC mask). Activity in both clusters was significantly stronger in BPD_N than in HC. Activity in left cuneus, lingual gyrus, and posterior cingulate was also significantly stronger in BPD_N than in BPD_D. In BPD_D, there was significantly stronger activity in left inferior frontal gyrus than in HC (Table 2).

\section{PPI analysis}

Significant group differences were observed for amygdala FC with clusters comprising bilateral fusiform gyrus, culmen, superior/medial frontal gyrus and middle frontal gyrus, right superior/middle temporal gyrus (insular cortex) and cingulate gyrus, left inferior parietal lobule (insular cortex) and anterior insula ( $p<0.05$, FWE-cluster-corrected), right middle occipital gyrus, and left claustrum (at $p<0.001$, uncorrected) (see Supplemental Table S1). HC showed (marginally) negative amygdala FC, while BPD groups showed positive amygdala FC with all of these regions. BPD_D differed from $\mathrm{HC}$ across all regions. BPD_N differed from $\mathrm{HC}$ regarding all regions except from middle occipital gyrus and superior temporal gyrus.

Compared to BPD_N, BPD_D showed reduced FC with left fusiform gyrus ( $t=2.07, p=0.048$, see Fig. 3a), while showing a stronger coupling between amygdala and left inferior parietal lobule ( $t=2.48, p=0.020)$, right superior/middle temporal gyrus $(t=2.20, p=0.036)$, and right middle occipital gyrus $(t=2.39, p=0.024$ ) (see Fig. 3b-d).

The MANCOVA with WM errors as covariate revealed similar results: compared to BPD_N, BPD_D showed a significantly stronger coupling between amygdala and left inferior parietal lobule $\left(F_{(1,26)}=5.96\right.$, $p=0.022)$, right superior/middle temporal gyrus $\left(F_{(1,26)}=2.54, p=0.046\right)$, and right middle occipital gyrus $\left(F_{(1,26)}=4.86, p=0.034\right)$, albeit group differences in amygdala $\mathrm{FC}$ with left fusiform gyrus were at a trend level $\left(F_{(1,26)}=2.25, p=0.063\right)$

\section{Discussion}

The aim of our study was to investigate the impact of dissociation on brain activity and amygdala functional connectivity (FC) during emotional distraction in the context of a delay-response WM task in un-medicated patients with BPD. Using script-driven imagery, dissociation was induced in 17 BPD patients ('BPD_D'), while 12 patients ('BPD_N') and 18 HC were exposed to neutral scripts. Afterwards, participants performed an Emotional Working Memory Task (EWMT) with negative vs. neutral interpersonal images versus no distractors. Main findings were:

- Behavioral performance Overall WM impairments (more incorrect responses and misses) in BPD_D compared to the other groups.

- Overall deactivation in the bilateral amygdala and diminished activity in the left cuneus, lingual gyrus, and posterior cingulate during emotional distraction in BPD_D compared to BPD_N; stronger left inferior frontal gyrus activity in BPD_D than in HC.

- Amygdala FC during negative vs. no distractors Increased amygdala connectivity with left inferior parietal lobule and right middle/superior temporal gyrus, but diminished amygdala FC with fusiform gyrus in BPD_D compared to the other groups.

The finding of impaired WM in BPD_D is consistent with previous research, pointing to detrimental effects of pathological dissociation on neuropsychological processes, such as learning, memory, attention, and interference inhibition [13, 53-55]. Since dissociation seems to influence neuropsychological functioning in BPD, dissociative symptoms should be taken into account in future experimental studies on affective-cognitive processing in $\mathrm{BPD}$, even when it is not the major focus of research.

Consistent with our previous studies [27, 28], the presentation of distractors in the EWMT elicited significant activity in brain regions implicated in emotion processing, attention, WM, and interference inhibition [10, 56]. During negative vs. no distractors, the two BPD groups showed different patterns of brain activity compared to HC: BPD_N patients exhibited increased activity in amygdala and insula as well as a hyper-connectivity of the amygdala, resembling previous neuroimaging findings in BPD [6-8].

Of note, BPD patients after dissociation induction did not differ significantly from $\mathrm{HC}$, while showing significantly less amygdala activity compared to BPD_N. As BPD groups were comparable regarding symptom severity, childhood trauma, PTSD comorbidity, anxiety, depressive mood, and basic working memory performance, findings point to a dampening effect of dissociation on amygdala reactivity, as proposed in current conceptualizations [15] [20].

During negative vs. no distractors, BPD_D further showed significantly lower activity in left cuneus, precuneus, and posterior cingulate-areas of the default mode 
Fig. 3 Results of the psychophysiological interaction analysis for functional connectivity (FC) of the bilateral amygdala (seed region of interest, depicted in green) during negative distractors versus no distraction in the context of the Emotional Working Memory Task in patients with borderline personality disorder (BPD) after dissociation induction (BPD_D) and after the neutral script (BPD_N) as well as in healthy controls (HC). The figure shows means \pm standard errors of the mean of parameter estimates for bilateral amygdala FC with a left fusiform gyrus, b left inferior parietal lobule, $\mathbf{c}$ right superior temporal gyrus, and $\mathbf{d}$ right middle occipital gyrus
A left fusiform gyrus [30, -55, -17]
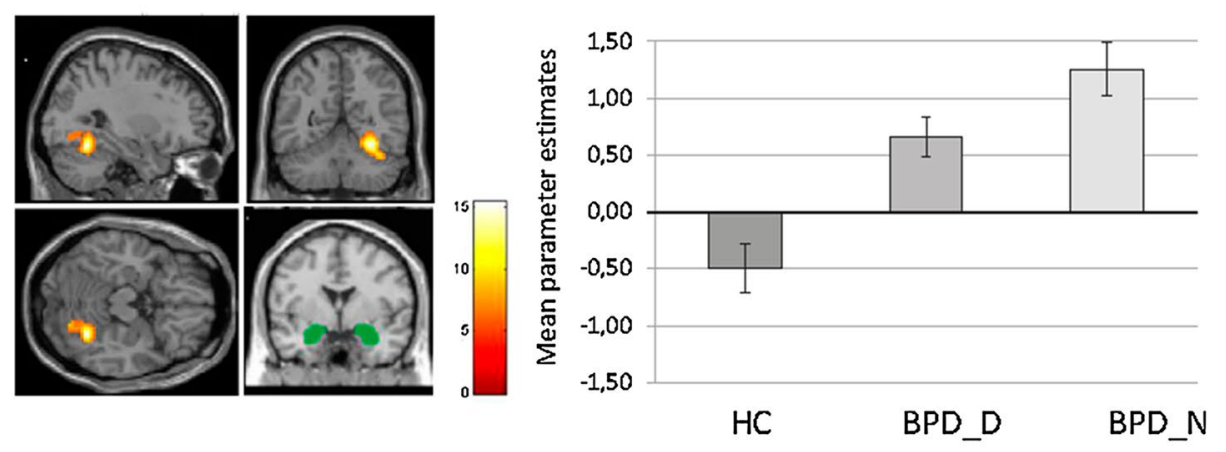

B left inferior parietal lobule $[-48,-40,25]$
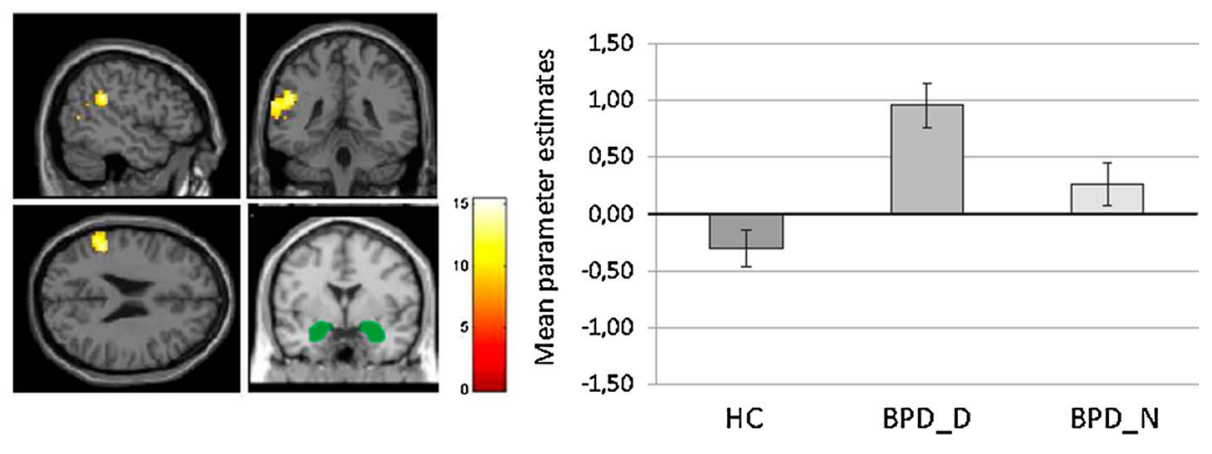

C right superior temporal gyrus $[54,-43,10]$
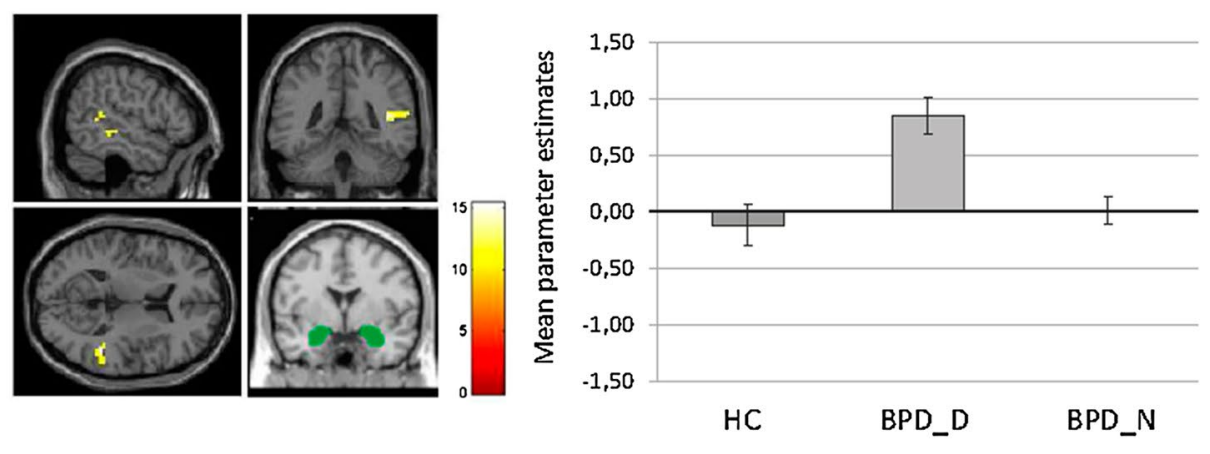

right middle occipital gyrus $[30,-76,22]$

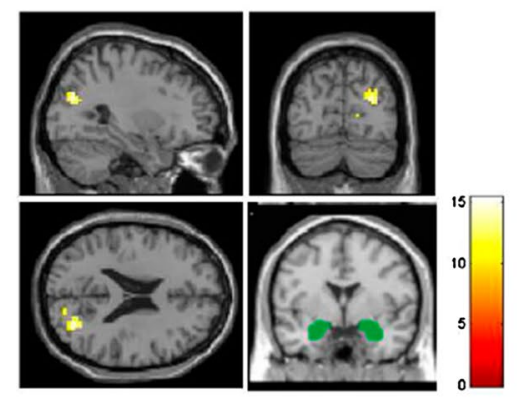

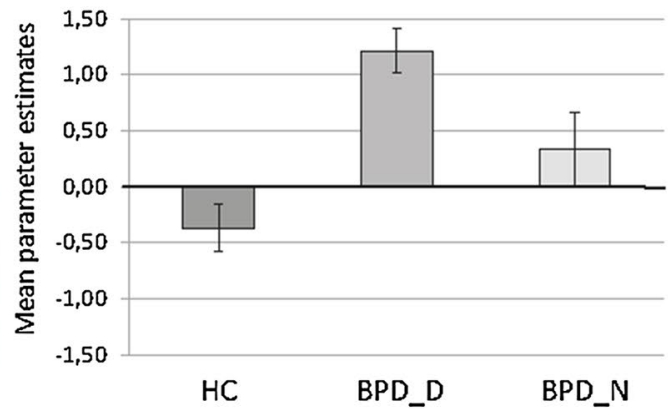

network that have been implicated in self-referential processing (e.g., autobiographical memory, rumination) [57-59]. Decreased activity in these regions may suggest reduced processing of task-irrelevant-but probably self-relevant-negative social material (reminders of interpersonal violence) in patients after dissociation induction.

Consistent with previous script-driven imagery studies [21, 29] and largely in line with our hypothesis, BPD_D 
patients showed stronger left inferior frontal gyrus activity than HC. However, no differences in MPFC and ACC were found and increased inferior frontal gyrus activity was not specific to BPD_D (i.e., also present in BPD_N). As BPD_N did not differ significantly from $\mathrm{HC}$ in WM, stronger recruitment of the inferior frontal gyrus in this group may reflect compensatory efforts to prevent the occurrence of interference disinhibition on a behavioral level [60, 61].

Extending previous research, we investigated how bilateral amygdala activity was correlated to activity in other brain areas during negative vs. no distractors. Both BPD groups differed significantly from $\mathrm{HC}$ in amygdala FC with frontal, temporal, occipital, and parietal areas. HC showed negative amygdala connectivity with these regions, resembling findings of previous fMRI studies using the EWMT or similar tasks [28, 62, 63], while BPD patients showed positive amygdala FC with these areas. Amygdala hyper-connectivity with frontal regions, including the ACC and $\mathrm{mPFC}$, was also observed in previous research and may reflect disturbed emotion processing in patients with BPD [64-69].

Importantly, we observed significant differences in amygdala connectivity between the two BPD groups, dependent on our experimental manipulation. Compared to the other groups, BPD patients exposed to the dissociation script showed diminished amygdala connectivity with left fusiform gyrus, which has been associated with encoding/processing of negative social material [70, 71]. BPD_D patients further showed a stronger coupling of the amygdala with clusters comprising right middle/superior temporal gyrus and left inferior parietal lobule. The superior temporal gyrus has previously been implicated in depersonalization and derealization [22-24] and is considered an important structure in a pathway including the amygdala and PFC, implicated in processing of language, social information, and self-perception [72]. In previous studies, higher self-reported dissociation was correlated to reduced gray matter volume [73] and increased activity in the middle/superior temporal gyrus [21] in BPD. The inferior parietal lobule has been implicated in emotion regulation and working memory-an increased information exchange of the amygdala with these areas may underlie altered emotional and self-referential processing during dissociation [74-78].

In summary, our neuroimaging findings suggest that a deactivation of the amygdala and altered interactions of this region with areas implicated in self-referential processing, cognitive control, visual perception, and sensory gating may contribute to dissociative states in BPD, while the precise mechanisms underlying stress-related dissociation remain elusive. More research is needed to clarify whether the neural patterns, observed in this study, reflect enhanced attempts to modulate states of arousal, as suggested by previous neuroimaging research in the dissociative subtype of PTSD [15] and models proposing that dissociation is a protective regulatory strategy in extremely stressful situations [17]. Dissociative responses may be an adaptive process when 'fight or flight' [83] is impossible [15, 17, 80], possibly stemming from an evolutionary older 'freezing system' [79-82]. However, the present findings provide further evidence that dissociation can become maladaptive by hindering a coherent processing of salient sensory, affective, and cognitive information in memory, which is crucial to a flexible adaptation to stressful situations [76-78]. Moreover, dissociation might not only dampen negative emotions but also positive emotions, which can have detrimental consequences for the quality of life and the maintenance of close relationships. Given these detrimental effects and previous findings of poor treatment outcome in BPD patients with pathological dissociative symptoms [18, 19, 84], our findings highlight the importance of taking dissociative symptoms into account when treating individuals with BPD.

To our knowledge, this is the first study in BPD revealing a significant impact of a dissociation induction on amygdala activity and functional connectivity during emotional distraction in the context of the EWMT. Present findings may shed a new light on stress-related dissociation in BPD, as affective-cognitive processing was studied both on a behavioral and neural level in an experimental setting which requires conscious manipulation of stressful (trauma-related) material in WM. Patient groups were matched regarding psychopathology and basic working memory and it was ensured that BPD_N patients were not dissociated. However, this led to a relatively small sample size and only female patients with a history of childhood abuse/neglect were included. We did not apply additional drug tests to rule out this possibility of false self-reports of our participants. Furthermore, it is likely that present findings may not be specific to BPD but also observable in other clinical populations with dissociative features, being a trans-diagnostic phenomenon [15, 75]. This means, more research with larger sample sizes, clinical control groups, and extended medical checks is needed to clarify whether the reported neural patterns can be replicated in other samples of BPD patients or are confounded by the afore-mentioned sample characteristics. As we used PPI, findings are restricted to our seed region and causality of interactions remains unknown [51, 52]. Tension ratings were significantly higher in BPD_D than BPD_N. Nevertheless, group differences in amygdala reactivity remained significant after including aversive tension as covariate.

All in all, our findings suggest a dampening effect of dissociation on activity in brain areas implicated in the processing of disturbing (trauma-related) information in BPD and 
an impairing effect on working memory, which plays a crucial role in goal-directed behavior. More research is needed to understand the impact of dissociation on other aspects of emotion regulation, cognition and identity in BPD and to gain more insight into this complex phenomenon.

Acknowledgements We thank all participants of this study for their collaboration.

\section{Compliance with ethical standards}

Conflict of interest None of the authors declares biomedical financial interests or potential conflicts of interest. Investigator B. M. Elzinga was funded by a VIDI grant by the Netherlands Organisation for Scientific Research (Grant Number 016·085·353).

Open Access This article is distributed under the terms of the Creative Commons Attribution 4.0 International License (http://creativecommons.org/licenses/by/4.0/), which permits unrestricted use, distribution, and reproduction in any medium, provided you give appropriate credit to the original author(s) and the source, provide a link to the Creative Commons license, and indicate if changes were made.

\section{References}

1. Crowell SE, Beauchaine TP, Linehan MM (2009) A biosocial developmental model of borderline personality: elaborating and extending Linehan's theory. Psychol Bull 135(3):495-510. doi:10.1037/a0015616

2. Schmahl C, Herpertz SC, Bertsch K et al (2014) Mechanisms of disturbed emotion processing and social interaction in borderline personality disorder: state of the art and research agenda of the German Clinical Research Unit. Borderline Personal Disord Emot Dysregulation 1:12. doi:10.1186/2051-6673-1-12

3. Banich MT, Mackiewicz KL, Depue BE, Whitmer A, Miller GA, Heller W (2009) Control mechanisms, emotion \& memory: a neural perspective with implications for psychopathology. Neurosci Biobehav Rev 33(5):613-630. doi:10.1016/jneubiorev.2008.09.010

4. Carpenter RW, Trull TJ (2013) Components of emotion dysregulation in borderline personality disorder: a review. Curr Psychiatry Rep 15:335. doi:10.1007/s11920-012-0335-2

5. Vermetten E, Spiegel D (2014) Trauma and dissociation: implications for borderline personality disorder. Curr Psychiatry Rep 16(2):434. doi:10.1007/s11920-013-0434-8

6. Krause-Utz A, Frost R, Winter D, Elzinga BM (2017) Dissociation and alterations in brain function and structure: implications for borderline personality disorder. Curr Psychiatry Rep 19(1):122. doi:10.1007/s11920-017-0757-y

7. Schulze L, Schmahl C, Niedtfeld I (2016) Neural correlates of disturbed emotion processing in borderline personality disorder: a multimodal meta-analysis. Biol Psychiat 79(2):97-106. doi:10.1016/j.biopsych.2015.03.027

8. Van Zutphen L, Siep N, Jacob GA, Goebel R, Arntz A (2015) Emotional sensitivity, emotion regulation and impulsivity in borderline personality disorder: a critical review of fMRI studies. Neurosci Biobehav Rev 51:64-76. doi:10.1016/j. neubiorev.2015.01.001

9. Ruocco AC, Amirthavasagam S, Choi-Kain LW, McMain SF (2013) Neural correlates of negative emotionality in borderline personality disorder: an activation-likelihood-estimation meta-analysis. Biol Psychiat 73(2):153-160. doi:10.1016/j. biopsych.2012.07.014

10. Phillips ML, Drevets WC, Rauch SL, Lane R (2003) Neurobiology of emotion perception I: the neural basis of normal emotion perception. Biol Psychiat 54(5):504-514. doi:10.1016/ S0006-3223(03)00171-9

11. Phillips ML, Sierra M (2003) Depersonalization disorder: a functional neuroanatomical perspective. Stress 6(3):157-165

12. Korzekwa MI, Dell PF, Pain C (2009) Dissociation and borderline personality disorder: an update for clinicians. Curr Psychiatry Rep 11(1):82-88. doi:10.1007/s11920-009-0013-1

13. Ebner-Priemer UW, Mauchnik J, Kleindienst N et al (2009) Emotional learning during dissociative states in borderline personality disorder. J Psychiatry Neurosci 34(3):214-222

14. Stiglmayr CE, Ebner-Priemer UW, Bretz J et al (2008) Dissociative symptoms are positively related to stress in borderline personality disorder. Acta Psychiatr Scand 117(2):139-147. doi:10.1111/j.1600-0447.2007.01126.x

15. Lanius RA, Vermetten E, Loewenstein RJ et al (2010) Emotion modulation in PTSD: clinical and neurobiological evidence for a dissociative subtype. Am J Psychiatry 167(6):640-647. doi:10.1176/appi.ajp.2009.09081168

16. American Psychiatric Association (2000) Diagnostic and statistical manual of mental disorders (4th ed., text rev.). American Psychiatric Association, Washington, DC. doi:10.1176/appi. books.9780890423349

17. Spiegel D, Loewenstein RJ, Lewis-Fernandez R et al (2011) Dissociative disorders in DSM-5. Depression and anxiety 28(12):E17-45. doi:10.1002/da.20923

18. Kleindienst N, Limberger MF, Ebner-Priemer UW et al (2011) Dissociation predicts poor response to dialectical behavioral therapy in female patients with borderline personality disorder. $\mathrm{J}$ Pers Disord 25(4):432-447. doi:10.1521/pedi.2011.25.4.432

19. Arntz A, Stupar-Rutenfrans S, Bloo J, van Dyck R, Spinhoven P (2015) Prediction of treatment discontinuation and recovery from borderline personality disorder: results from an RCT comparing schema therapy and transference focused psychotherapy. Behav Res Ther 74:60-71. doi:10.1016/j. brat.2015.09.002

20. Sierra M, Berrios GE (1998) Depersonalization: neurobiological perspectives. Biol Psychiat 44(9):898-908. doi:10.1016/ S0006-3223(98)00015-8

21. Ludaescher P, Valerius G, Stiglmayr C et al (2010) Pain sensitivity and neural processing during dissociative states in patients with borderline personality disorder with and without comorbid posttraumatic stress disorder: a pilot study. J Psychiatry Neurosci 35(3):177-184. doi:10.1503/jpn.090022

22. Simeon D, Guralnik O, Hazlett EA, Spiegel-Cohen J, Hollander E, Buchsbaum MS (2000) Feeling unreal: a PET study of depersonalization disorder. Am J Psychiatry 157(11):17821788. doi:10.1176/appi.ajp.157.11.1782

23. Lanius RA, Williamson PC, Bluhm RL et al (2005) Functional connectivity of dissociative responses in posttraumatic stress disorder: a functional magnetic resonance imaging investigation. Biol Psychiat 57(8):873-884. doi:10.1016/j. biopsych.2005.01.011

24. Stein JL, Wiedholz LM, Bassett DS et al (2007) A validated network of effective amygdala connectivity. Neuroimage 36(3):736745. doi:10.1016/j.neuroimage.2007.03.022

25. Roy AK, Shehzad Z, Margulies DS et al (2009) Functional Connectivity of the human amygdala using resting state fMRI. NeuroImage 45(2):614-626. doi:10.1016/j. neuroimage.2008.11.030

26. Hazlett EW, Zhang J, New AS et al (2012) Potentiated amygdala response to repeated emotional pictures in 
borderline personality disorder. Biol Psychiat 72(6):448-456. doi:10.1016/j.biopsych.2012.03.027

27. Krause-Utz A, Oei NY, Niedtfeld I et al (2012) Influence of emotional distraction on working memory performance in borderline personality disorder. Psychol Med 42(10):2181-2192. doi:10.1017/S0033291712000153

28. Krause-Utz A, Elzinga BM, Oei NY et al (2014) Amygdala and dorsal anterior cingulate connectivity during an emotional working memory task in borderline personality disorder patients with interpersonal trauma history. Front Hum Neurosci 8:848. doi:10.3389/fnhum.2014.00848

29. Winter D, Krause-Utz A, Lis S et al (2015) Dissociation in borderline personality disorder: disturbed cognitive and emotional inhibition and its neural correlates. Psychiatry Res Neuroimaging 233(3):339-351. doi:10.1016/j.pscychresns.2015.05.018

30. Lang PJ, Bradley MM, Cuthbert BN (2005) International Affective Picture System (IAPS): digitized photographs, instruction manual and affective ratings (Technical Report A, $6^{\text {th }}$ Edn). University of Florida, Gainesville

31. Stiglmayr C, Schmahl C, Bremner JD, Bohus M, Ebner-Priemer U (2009) Development and psychometric characteristics of the DSS-4 as a short instrument to assess dissociative experience during neuropsychological experiments. Psychopathology 42(6):370-374. doi:10.1159/000236908

32. First MB, Spitzer RL, Gibbon M, Williams JB (1997) Structured Clinical Interview for DSM-IV Axis I Disorders-Clinical Version (SCID-CV). American Psychiatric Press, Washington, DC

33. Loranger AW (1999) International Personality Disorder Examination (IPDE): DSM-IV and ICD-10 modules. Psychological Assessment Resources, Odessa

34. Bohus M, Kleindienst N, Limberger MF et al (2009) The short version of the Borderline Symptom List (BSL-23): development and initial data on psychometric properties. Psychopathology 42(1):32-39. doi:10.1159/000173701

35. Bernstein DP, Stein JA, Newcomb MD et al (2003) Development and validation of a brief screening version of the Childhood Trauma Questionnaire. Child Abuse Negl 27(2):169-190. doi:10.1016/S0145-2134(02)00541-0

36. Bernstein EM, Putnam FW (1986) Development, reliability, and validity of a dissociation scale. J Nerv Ment Dis 174(12):727735. doi:10.1097/00005053-19861200000004

37. Beck AT, Steer RA, Brown GK (1996) Manual for the Beck depression inventory-II. Psychological Corporation, San Antonio

38. Spielberger CD, Gorsuch RL, Lushene RE (1970) Manual for the state-trait anxiety inventory. Consulting Psychologists Press, Palo Alto

39. Ward MF, Wender PH, Reimherr FW (1993) The Wender Utah Rating Scale: an aid in the retrospective diagnosis of childhood attention deficit hyperactivity disorder. Am J Psychiatry 150(6):885-890. doi:10.1176/ajp.150.6.885

40. Roesler M, Retz-Junginger P, Retz W, Stieglitz RD (2008) [Homburger ADHS-Skalen für Erwachsene (HASE)]. Manual [in German]. Hogrefe, Göttingen

41. Sternberg S (1966) High-speed scanning in human memory. Science 153(3736):652-654. doi:10.1126/ science. 153.3736 .652

42. Oei NY, Tollenaar MS, Spinhoven P, Elzinga BM (2009) Hydrocortisone reduces emotional distracter interference in working memory. Psychoneuroendocrinology 34(9):12841293. doi:10.1016/j.psyneuen.2009.03.015

43. Oei NY, Veer IM, Wolf OT, Spinhoven P, Rombouts SA, Elzinga BM (2012) Stress shifts brain activation towards ventral "affective" areas during emotional distraction. Soc Cogn Affect Neurosci 7(4):403-412. doi:10.1093/scan/nsr024
44. Friston KJ, Frith CD, Turner R, Frackowiak RS (1995) Characterizing evoked hemodynamics with fMRI. Neuroimage 2(2):157-165. doi:10.1006/nimg. 1995.1018

45. Tzourio-Mazoyer N, Landeau B, Papathanassiou D et al (2002) Automated anatomical labeling of activations in SPM using a macroscopic anatomical parcellation of the MNI MRI single-subject brain. NeuroImage 15(1):273-289. doi:10.1006/ nimg.2001.0978

46. Gläscher J (2009) Visualization of group inference data in functional neuroimaging. Neuroinformatics 7(1):73-82. doi:10.1007/s12021-008-9042-x

47. Donegan NH, Sanislow CA, Blumenberg HP et al (2003) Amygdala hyperreactivity in borderline personality disorder: implications for emotional dysregulation. Biol Psychiat 54(11):1284-1293. doi:10.1016/S0006-3223(03)00636-X43

48. Niedtfeld I, Schulze L, Kirsch P, Herpertz SC, Bohus M, Schmahl C (2010) Affect regulation and pain in borderline personality disorder: a possible link to the understanding of self-injury. Biol Psychiatry 68(4):383-391. doi:10.1016/j. biopsych.2010.04.015

49. Schulze L, Domes G, Krüger A et al (2011) Neuronal correlates of cognitive reappraisal in borderline patients with affective instability. Biol Psychiatry 69(6):564-573. doi:10.1016/j. biopsych.2010.10.025

50. McLaren DG, Ries ML, Xu G, Johnson SC (2012) A generalized form of context-dependent psychophysiological interactions (gPPI): a comparison to standard approaches. NeuroImage 61(4):1277-1286. doi:10.1016/j.neuroimage.2012.03.068

51. Friston KJ, Büchel C, Fink GR, Morris J, Rolls E, Dolan RJ (1997) Psychophysiological and modulatory interactions in neuroimaging. Neuroimage 6(3):218-229. doi:10.1006/ nimg.1997.0291

52. O'Reilly JX, Woolrich MW, Behrens TE, Smith SM, JohansenBerg H (2012) Tools of the trade: psychophysiological interactions and functional connectivity. Soc Cogn Affect Neurosci 7(5):604-609. doi:10.1093/scan/nss055

53. Chiu CD, Yeh YY, Huang CL, Wu YC, Chiu YC, Lin CC (2010) Unintentional memory inhibition is weakened in nonclinical dissociators. J Behav Ther Exp Psychiatry 41(2):117124. doi:10.1016/j.jbtep.2009.11.003

54. Haaland VØ, Landrø NI (2009) Pathological dissociation and neuropsychological functioning in borderline personality disorder. Acta Psychiatr Scand 119(5):383-392. doi:10.1111/j.1600-0447.2008.01323.x

55. Winter D, Elzinga B, Schmahl C (2014) Emotions and memory in borderline personality disorder. Psychopathology 47(2):7185. doi: $10.1159 / 000356360$

56. Ochsner KN, Gross JJ (2007) The neural architecture of emotion regulation. In: Gross JJ, Buck R (eds) The Handbook of emotion regulation. Guilford Press, New York, pp 87-109

57. Raichle ME, MacLeod AM, Snyder AZ, Powers WJ, Gusnard DA, Shulman GL (2001) A default mode of brain function. Proc Natl Acad Sci USA 98(2):676-682. doi:10.1073/ pnas.98.2.676

58. Buckner R, Andrews-Hanna J, Schacter D (2008) The brain's default network: anatomy, function, and relevance to disease. Ann N Y Acad Sci 1124:1-38. doi:10.1196/annals.1440.011

59. Menon V (2011) Large-scale brain networks and psychopathology: a unifying triple network model. Trends Cogn Sci 15(10):483-506. doi:10.1016/j.tics.2011.08.003

60. Jacob GA, Zvonik K, Kamphausen S et al (2013) Emotional modulation of motor response inhibition in women with borderline personality disorder: an fMRI study. J Psychiatry Neurosci 38(3):164-172. doi:10.1503/jpn.120029

61. Van Eijk J, Sebastian A, Krause-Utz A et al (2015) Women with borderline personality disorder do not show altered 
BOLD responses during response inhibition. Psychiatry Res 234(3):378-389. doi:10.1016/j.pscychresns.2015.09.017

62. Anticevic A, Repovs G, Barch DM (2010) Resisting emotional interference: brain regions facilitating working memory performance during negative distraction. Cogn Affect Behav Neurosci 10(2):159-173. doi:10.3758/CABN.10.2.159

63. Mitchell DG, Luo Q, Mondillo K, Vythilingam M, Finger EC, Blair RJ (2008) The interference of operant task performance by emotional distracters: an antagonistic relationship between the amygdala and frontoparietal cortices. NeuroImage 40(2):859868. doi:10.1016/j.neuroimage.2007.08.002

64. Cullen KR, Vizueta N, Thomas KM et al (2011) Amygdala functional connectivity in young women with borderline personality disorder. Brain Connect 1(1):61-71. doi:10.1089/ brain.2010.0001

65. Kamphausen S, Schroder P, Maier S et al (2013) Medial prefrontal dysfunction and prolonged amygdala response during instructed fear processing in borderline personality disorder. World J Biol Psychiatry 14(4):307-318, S1-4. doi:10.3109/156 22975.2012.665174

66. Niedtfeld I, Kirsch P, Schulze L, Herpertz SC, Bohus M, Schmahl C (2012) Functional connectivity of pain-mediated affect regulation in borderline personality disorder. PLoS One 7(3):e33293. doi:10.1371/journal.pone.0033293

67. Koenigsberg HW, Denny BT, Fan J et al (2014) The neural correlates of anomalous habituation to negative emotional pictures in borderline and avoidant personality disorder patients. Am J Psychiatry 171(1):82-90. doi:10.1176/appi.ajp.2013.13070852

68. Krause-Utz A, Veer IM, Rombouts SARB, Bohus M, Schmahl C, Elzinga BM (2014) Amygdala and anterior cingulate restingstate functional connectivity in borderline personality disorder patients with a history of interpersonal trauma. Psychol Med 44(13):2889-2901. doi:10.1017/S0033291714000324

69. Salvador R, Vega D, Pascual JC et al (2016) Converging medial frontal resting state and diffusion-based abnormalities in borderline personality disorder. Biol Psychiat 79(2):107-116. doi:10.1016/j.biopsych.2014.08.026

70. Kruschwitz JD, Meyer-Lindenberg A, Veer IM et al (2015) Segregation of face sensitive areas within the fusiform gyrus using global signal regression? A study on amygdala resting-state functional connectivity. Hum Brain Mapp 36(10):4089-40103. doi: $10.1002 / \mathrm{hbm} .22900$

71. Molapour T, Golkar A, Navarrete CD, Haaker J, Olsson A (2015) Neural correlates of biased social fear learning and interaction in an intergroup context. NeuroImage 121:171-183. doi:10.1016/j. neuroimage.2015.07.015

72. Bigler ED, Mortensen S, Neeley ES et al (2007) Superior temporal gyrus, language function, and autism. Dev Neuropsychol 31(2):217-238. doi:10.1080/87565640701190841
73. Niedtfeld I, Schulze L, Krause-Utz A, Demirakca T, Bohus M, Schmahl C (2013) Voxel-based morphometry in women with borderline personality disorder with and without comorbid posttraumatic stress disorder. PLoS One 8(6):e65824. doi:10.1371/ journal.pone.0065824

74. Nicholson AA, Densmore M, Frewen PA et al (2015) The dissociative subtype of posttraumatic stress disorder: unique restingstate functional connectivity of basolateral and centromedial amygdala complexes. Neuropsychopharmacology 40(10):23172326. doi:10.1038/npp.2015.79

75. Lanius RA (2015) Trauma-related dissociation and altered states of consciousness: a call for clinical, treatment, and neuroscience research. Eur J Psychotraumatol 6:27905. doi:10.3402/ejpt. v6.27905

76. Bremner JD, Vermetten E, Southwick SM, Krystal JH, Charney DS (1998) Trauma, memory, and dissociation: an integrative formulation. American Psychiatric Association Press, Washington, DC, pp 365-402

77. Conway MA, Pleydell-Pearce CW (2000) The construction of autobiographical memories in the self-memory system. Psychol Rev 107(2):261-288. doi:10.1037/0033-295X.107.2.261

78. Rueda MR, Posner MI, Rothbart MK (2005) The development of executive attention: contributions to the emergence of selfregulation. Dev Neuropsychol 28(2):573-594. doi:10.1207/ s15326942dn2802_2

79. Fanselow MS, Lester LS (1988) A functional behavioristic approach to aversively motivated behavior: predatory imminence as a determinant of the topography of the defensive behavior. In: Bolles RC, Breecher MD (eds) Evolution and learning. Erlbaum, Hilsdale, pp 185-212

80. Schauer M, Elbert T (2010) Dissociation following traumatic stress: etiology and treatment. J Psychol 218(2):109-127. doi:10.1027/0044-3409/a000018

81. Zelikowsky M, Hersman S, Chawla MK, Barnes CA, Fanselow MS (2014) Neuronal ensembles in amygdala, hippocampus, and prefrontal cortex track differential components of contextual fear. J Neurosci 34(25):8462-8466. doi:10.1523/ JNEUROSCI.3624-13.2014

82. Hagenaars MA, Oitzl M, Roelofs K (2014) Updating freeze: aligning animal and human research. Neurosci Biobehav Rev 47:165-176. doi:10.1016/j.neubiorev.2014.07.021

83. Cannon WB (1929) Bodily changes in pain, hunger, fear, and range. Appleton-Century-Crofts, New York

84. Spitzer C, Barnow S, Freyberger HJ, Grabe HJ (2007) Dissociation predicts symptom-related treatment outcome in short-term inpatient psychotherapy. Aust N Z J Psychiatry 41(8):682-687. doi:10.1080/00048670701449146 